\title{
PROSPECTS OF USING WALNUT IN TECHNOLOGIES OF DRINKS
}

\author{
Inna TIURIKOVA*1, Mykhailo PERESICHNYI** \\ *Higher Educational Institution of Ukoopspilka "Poltava University of \\ Economics and Trade”, 3 Koval str., 36014, Poltava, Ukraine \\ ${ }^{* *}$ National University of Food Technologies, 68 Volodymyrska str., \\ 01601, Kyiv, Ukraine
}

\begin{abstract}
The results of studies in the field of beverage functionality using walnut are presented. The main components such as celery, Jerusalem artichokes, carrots, pumpkin and rhubarb, as a dietary supplement - extracts from walnut of milk-maturity stage are offered for creating blends. The basic physical and chemical properties of fruit and vegetable raw materials and semi-finished products created on the base of them have been studied, and their nutritional and biological value has been proved. Rational technologies of fruit and vegetable blends with nut additives have been identified. Their biological value has been confirmed. Drinks are recommended for use in the daily diet of human beings to satisfy thirst and enrich the body by biologically valuable components.
\end{abstract}

Keywords: walnuts, milk-wax maturity stage, extracts, biologically active substances, drinks

\section{INTRODUCTION}

The way of life in modern conditions differs from the lifestyle of previous generations. Technological advances and the transition from the industrial age to the information age have accelerated the pace of life, created additional pressure on human beings, and changed their entire activity. Therefore, more and more people tend to lead a healthy style of life.

Of particular relevance is the creation of a new generation of food, because of the insufficient supply of vital nutrients (vitamins, minerals, amino acids, dietary fiber, etc.) in the diet of population. Their deficiency is observed in representatives of all population groups (Peresichnyi et al,2012).

\footnotetext{
${ }^{1}$ Corresponding author. Mailing address: Higher Educational Institution of Ukoopspilka "Poltava University of Economics and Trade", 3 Koval str., 36014, Poltava, Ukraine. Tel: + 38 0509582693. E-mail: inna-tyurikova@yandex.ru
} 
The basis of competitive innovative functional food should be formed by highly professional fundamental complex research and industrial testing. The products of high biological value are created by combining, i.e. adding biologically active additives (BAA) in consumer foods, such as dairy products, confectionery, soft and alcoholic drinks. Traditionally such additives as juices, extracts, extracts from fruits and berries, medicinal plants are used. Adding supplements can regulate the chemical and mineral composition of foods that means to get food with prophylactic and health care properties (Tiurikova et al., 2008).

One way to improve food quality of foods and to improve the structure of nutrition of the population is to introduce new types of biologically valuable plant materials.

Walnut is a unique plant, in which all parts contain many bioactive substances: bark contains triterpenoids, steroids, alkaloids, vitamin C, tannins, quinones (yukhlon and etc.); leaves contain aldehydes, essential oils, alkaloids, vitamins C, PP, carotene, phenolcarbonic acids, tannins, coumarins, flavonoids, anthocyanins, quinones and high aromatic hydrocarbons; pericarp contains organic acids, vitamin $\mathrm{C}$, carotene, phenolcarbonic acids, tannins, coumarins and quinones. In green nuts vitamins $\mathrm{C}, \mathrm{B}_{1}, \mathrm{~B}_{2}, \mathrm{PP}$, carotene and quinines can be found, in ripe nuts sitosterol, vitamin $\mathrm{C}, \mathrm{B}_{1}, \mathrm{~B}_{2}, \mathrm{PP}$, carotene, tannins, quinones and fatty oil, consisting of linoleic, linolenic, oleic, palmitic acid and others, as well as fiber, iron and cobalt salts. Leaves, green fruits and pericarp differ (compared to other plants) by record concentration of vitamin C, P-active polyphenols and iodine. However, leaves and green nuts are widely used in folk medicine and pharmacology as well as green nuts are used in the food industry - for the production of jams, liqueurs (Tiurikova et al., 2008) (Tiurikova and Kholodnyi, 2008).

Drinks are an important part of the overall nutrition scheme. The range of drinks is presented solely by drinks produced with the use of traditional raw materials such as fruits and vegetables. The development and introduction of new technologies, identification of food compositions using uncommon plant materials such as walnut of milk-wax maturity stage is relevant.

\section{MATERIALS AND METHODS}

During the studies standardized methods were used. In raw materials, semifinished products, beverages the key physical, chemical and organoleptic properties were defined.

Sampling was carried out according to the requirements of GOST $26313-84$, preparation of samples - according to GOST 26671-85. In determining the parameters the following documents were used: 
- content of dry substances in the raw material - GOST 28561-90;

- mass fraction of soluble solids - refractometric method of DSTU ISO 2173: 2007;

- $\quad$ active acidity $(\mathrm{pH})$ - potentiometric method of DSTU EN 1132: 2005;

- mass fraction of titrated acids (in terms of malic or citric acid) volumetric titration method EN 12147-2003;

- the total content of phenolic compounds - by Folin-Chocalteu in terms of gallic acid per GOST 3845-99;

- vitamin C content - by iodometric method according to GOST 24555689 ;

- content of pectin - by Ca-pectat method (Arasymovych, 1990);

- content of protopectin - GOST 29059-91;

- organoleptic assessment - GOST 8756.1-79.

All determinations were carried out in two or three replications and for getting the final result arithmetic average was calculated. To obtain comparative data, analysis were carried out in all the same conditions.

\section{RESULTS AND DISCUSSIONS}

Thorough research allowed to determine optimal timing of industrial raw nut harvesting namely fruits of milk-wax maturity stage - mid- and late June; pericarp of ripe nuts - late September and early October.

The influence of complex technological factors on extraction of biologically active substances of nuts (the type and concentration of extractant, hydrological values, duration and frequency rate of extraction, particle size material) has been identified. The technology of extract of walnut of milkwax maturity stage by double extraction has been developed. It was identified that the maximum extraction of bioactive components from raw materials is possible by extraction with $70 \%$ aqueous alcohol solution, the hydro ratio of raw materials: extractant $1: 1$ or $50 \%$ aqueous sugar solution under hydro ratio - 1:0.75, particle size of raw material $-10 \ldots 15 \mathrm{~mm}$, duration - no more than 30 days (Tiurikova, 2012) (Tiurikova, 2013).

The components selected for drinks were not common in the field of soft drinks vegetable raw materials - celery, Jerusalem artichoke, carrots, pumpkin, and rhubarb.

$100 \mathrm{~g}$ of celery root contains $320 \mathrm{mg}$ of potassium, $80 \mathrm{mg}$ of phosphorus, 68 $\mathrm{mg}$ of calcium, $9 \mathrm{mg}$ of magnesium, $0.53 \mathrm{mg}$ of iron, $0.31 \mathrm{mg}$ of zinc, 0.15 $\mathrm{mg}$ of manganese and sodium. Scientists have found apigenin in celery - a flavonoid which, in their opinion, can stop the development of certain cancer cells. Traditional medicine advised to use celery juice to improve appetite and digestion, as a diuretic and analgesic remedy in treating diseases such as anemia, arthritis, thrombophlebitis, blood diseases, metabolism, 
atherosclerosis, hypertension, diabetes, gout, obesity, cystitis, edema, and even migraines, nervousness and insomnia (Tiurikova, 2013).

Jerusalem artichoke contains potassium, calcium, silicon, magnesium, sodium, chromium, zinc, iron, phosphorus, iodine, fiber, protein, essential amino acids, a large number of pectin. This product is rich in vitamins B1, B2, B6, B9, A, C, PP. Jerusalem artichoke juice is used for strong heartburn, for treatment of tachycardia, coronary heart disease, and hypertension. But the main value for the human body belongs to inulin and inulins (in tubers $14 \ldots 22 \%$ ), which is an effective agent in the treatment of atherosclerosis, diabetes, obesity, various intoxications. Due to a set of biologically active substances it positively affects the function of many organs and systems, has anti-sclerotic effect, improves vascular properties and rheological parameters of blood, and strengthens the immune system. Jerusalem artichoke is considered to be a valuable component of dietary and nutritional therapy (Sarnitsky, 2007).

Rhubarb is an important raw material to be added to the human diet. The peculiarity of the chemical composition of rhubarb is a significant set of organic acids such as malic, citric $(1.58 \ldots 2.6 \%)$, succinic acid, acetic acid, oxalic acid $(0.14 \ldots 0.25 \%)$ and others. Petioles contain sugar and $2.5 \%$, of organic acids, about $3.5 \%$ of vitamin C (30 mg / $100 \mathrm{~g})$, B, PP, E, carotene, pectin $(0.8 \%)$, nitrogenous substances $(1.1 \%)$, mineral salts, potassium, calcium, phosphorus, magnesium (Tiurikova, 2013).

Carrots contain a significant amount of $\beta$-carotene, vitamins B1, B2, B6, C, $\mathrm{E}, \mathrm{PP}$ and other biologically active substances. Complex sugars and minerals makes carrots delicious, nutritious diet product. Its roots contain small amounts of biologically essential amino acids, pectin, protein, essential oil which gives specific carrot odor. Carrot juice is a natural source of vitamin A and carotene as well as phosphorus, calcium and iron - all these things are necessary in order to stay young for a long time (Tiurikova, 2013).

Pumpkin is rich in sugars, carotene, vitamins B1, B2, B5, B6, E, PP, and very rare vitamin $\mathrm{T}$, which helps to accelerate metabolism, blood clotting and platelet formation; in vitamin $\mathrm{K}$ which is necessary for blood clotting process; in minerals, including potassium, calcium, iron, etc ; in pectin which promotes excretion of toxic substances and cholesterol. Pumpkin contains $90 \%$ of the structured water, which adjusts the body's cells to a harmonious existence (Sarnitsky, 2007).

Physical and chemical characteristics of fruit and vegetable raw materials selected to create drinks with addition of walnut extract are presented in Tables 1 and 2 .

To create drinks from Jerusalem artichoke blending of traditional raw fruit plum, apple, lemon, orange, Cornelian cherry was carried out. It is proved that the fruits of Jerusalem artichoke, plums and apples in a state of full technical 
maturity and primary consumer maturity contain small amounts of vitamins compared with lemon, orange and Cornelian cherry, but rich in pectin and carbohydrates (Table 1).

Table 1. Physical and chemical characteristics of fruit and vegetable raw materials

\begin{tabular}{|l|c|c|c|c|c|c|}
\hline \multirow{2}{*}{ Raw material } & \multicolumn{3}{|c|}{ Mass content, \% } & \multicolumn{2}{c|}{$\begin{array}{c}\text { Content by weight, } \\
\mathrm{mg} / 100 \mathrm{~g}\end{array}$} & \multirow{2}{*}{\begin{tabular}{c}
$\mathrm{pH}$ \\
\cline { 2 - 6 }
\end{tabular}} \\
\cline { 2 - 6 } & $\begin{array}{c}\text { dry } \\
\text { substances }\end{array}$ & $\begin{array}{c}\text { titrated } \\
\text { acids }\end{array}$ & $\begin{array}{c}\text { pectin } \\
\text { substances }\end{array}$ & $\begin{array}{c}\text { L-anti- } \\
\text { scorbutin }\end{array}$ & $\begin{array}{c}\text { polyphe- } \\
\text { nols }\end{array}$ & \\
\hline $\begin{array}{l}\text { Jerusalem } \\
\text { artichoke raw }\end{array}$ & $\begin{array}{c}18.4 / 17.4 \\
\pm 0.8\end{array}$ & $\begin{array}{c}0.28 / 0.42 \\
\pm 0.02\end{array}$ & $\begin{array}{c}1.840 \pm 0.04 \\
/ 0.52 \pm 0.02\end{array}$ & $\begin{array}{c}7.04 / 6.35 \\
\pm 0.03\end{array}$ & $20 \pm 0.9$ & $\begin{array}{c}6.20 \\
\pm 0.3\end{array}$ \\
\hline $\begin{array}{l}\text { Cornelian } \\
\text { cherry raw }\end{array}$ & $20.6 \pm 0.8$ & $1.25 \pm 0.04$ & $0.92 \pm 0.04$ & $35.90 \pm 2$ & $\begin{array}{c}6200 \pm 3 \\
10\end{array}$ & $\begin{array}{c}3.1 \\
\pm 0.15\end{array}$ \\
\hline $\begin{array}{l}\text { Plum } \\
\text { raw }\end{array}$ & $17.2 \pm 0.8$ & $1.17 \pm 0.04$ & $5.336 \pm 0.25$ & $1.14 \pm 0.04$ & $430 \pm 20$ & $\begin{array}{c}3.05 \\
\pm 0.15\end{array}$ \\
\hline $\begin{array}{l}\text { Apple } \\
\text { raw }\end{array}$ & $13.0 \pm 0.5$ & $0.43 \pm 0.02$ & $3.128 \pm 0.12$ & $3.52 \pm 0.02$ & $\begin{array}{c}\text { were not } \\
\text { identified }\end{array}$ & $\begin{array}{c}3.10 \\
\pm 0.15\end{array}$ \\
\hline $\begin{array}{l}\text { Lemon } \\
\text { raw }\end{array}$ & $8.0 \pm 0.3$ & $6.03 \pm 0.2$ & $0.331 \pm 0.01$ & $23.76 \pm 1.0$ & $\begin{array}{c}\text { were not } \\
\text { identified }\end{array}$ & $\begin{array}{c}2.05 \\
\pm 0.10\end{array}$ \\
\hline $\begin{array}{l}\text { Orange } \\
\text { raw }\end{array}$ & $8.6 \pm 0.3$ & $1.47 \pm 0.04$ & $0.349 \pm 0.12$ & $22.88 \pm 1.0$ & $\begin{array}{c}\text { were not } \\
\text { identified }\end{array}$ & $\begin{array}{c}2.85 \\
\pm 0.15\end{array}$ \\
\hline $\begin{array}{l}\text { Pureed } \\
\text { cornelian } \\
\text { cherry }\end{array}$ & $20.2 \pm 0.8$ & $1.23 \pm 0.02$ & $0.85 \pm 0.03$ & $30.16 \pm 1.2$ & $1350 \pm 60$ & $\begin{array}{c}3.4 \\
\pm 0.15\end{array}$ \\
\hline $\begin{array}{l}\text { Pureed } \\
\text { Jerusalem } \\
\text { artichoke }\end{array}$ & $16.3 \pm 0.07$ & $0.48 \pm 0.02$ & $0.46 \pm 0.02$ & $4.87 \pm 0.2$ & $\begin{array}{c}\text { were not } \\
\text { identified }\end{array}$ & $\begin{array}{c}6.15 \\
\pm 0.3\end{array}$ \\
\hline Celery juice & $8.00 \pm 0.4$ & $1.2 \pm 0.05$ & $1.3 \pm 0.02$ & $4.40 \pm 0.2$ & $\begin{array}{c}\text { were not } \\
\text { identified }\end{array}$ & $\begin{array}{c}6.00 \\
\pm 0.2\end{array}$ \\
\hline $\begin{array}{l}\text { Rhubarb juice } \\
\text { unclarified }\end{array}$ & $3.4 \pm 0.15$ & $1.40 \pm 0.05$ & $0.320 \pm 0.01$ & $4.9 \pm 0.2$ & $\begin{array}{c}\text { were not } \\
\text { identified }\end{array}$ & $\begin{array}{c}3.30 \\
\pm 0.1\end{array}$ \\
\hline
\end{tabular}

Recipes of drinks on the basis of celery juice with adding of water-alcohol extract of walnut, honey or sugar syrup have been developed. Technologies of drinks and their physical and chemical characteristics are given in Table 3. It was established that the selected recipe correlation of food components allowed us to create unique drinks based on celery juice with indicators higher than source raw material as to the content of sugars, organic acids and ascorbic acid (Tables 1 - 3). 
Table 2. Physical and chemical characteristics of carotene containing vegetable raw materials

\begin{tabular}{|l|c|c|c|c|c|c|c|}
\hline \multirow{2}{*}{$\begin{array}{l}\text { Raw } \\
\text { material }\end{array}$} & \multicolumn{4}{|c|}{ Mass content, \% } & \multicolumn{2}{c|}{$\begin{array}{c}\text { Content by } \\
\text { weight, mg/100 g }\end{array}$} & \multirow{2}{*}{ pH } \\
\cline { 2 - 7 } & \multicolumn{2}{|c|}{ dry substances } & titrated & $\begin{array}{c}\text { pectin } \\
\text { sub- } \\
\text { acids } \\
\text { tometric }\end{array}$ & $\begin{array}{c}\text { L-anti- } \\
\text { stances }\end{array}$ & $\begin{array}{c}\beta- \\
\text { scorbutin } \\
\text { carotin }\end{array}$ & \\
\hline $\begin{array}{l}\text { Carrots } \\
\text { raw }\end{array}$ & - & $8.40 \pm 0.4$ & $\begin{array}{c}0.180 \\
\pm 0.01\end{array}$ & $\begin{array}{c}0.460 \\
\pm 0.02\end{array}$ & $\begin{array}{c}4.9 \\
\pm 0.2\end{array}$ & $\begin{array}{c}3.75 \\
\pm 0.15\end{array}$ & $\begin{array}{c}6.10 \\
\pm 0.3\end{array}$ \\
\hline $\begin{array}{l}\text { Carrot } \\
\text { juice } \\
\text { unclarified }\end{array}$ & $8.3 \pm 0.4$ & - & $\begin{array}{c}0.174 \\
\pm 0.01\end{array}$ & $\begin{array}{c}0.515 \\
\pm 0.02\end{array}$ & $\begin{array}{c}4.7 \\
\pm 0.2\end{array}$ & $\begin{array}{c}3.53 \\
\pm 0.15\end{array}$ & $\begin{array}{c}6.55 \\
\pm 0.3\end{array}$ \\
\hline $\begin{array}{l}\text { Pureed } \\
\text { carrots }\end{array}$ & $8.6 \pm 0.4$ & $8.12 \pm 0.4$ & $\begin{array}{c}0.110 \\
\pm 0.01\end{array}$ & $\begin{array}{c}0.552 \\
\pm 0.02\end{array}$ & $\begin{array}{c}3.4 \\
\pm 0.15\end{array}$ & $\begin{array}{c}2.69 \\
\pm 0.10\end{array}$ & $\begin{array}{c}6.10 \\
\pm 0.3\end{array}$ \\
\hline $\begin{array}{l}\text { Pumpkin } \\
\text { raw }\end{array}$ & $5.5 \pm 0.2$ & $6.0 \pm 0.3$ & $\begin{array}{c}0.027 \\
\pm 0.001\end{array}$ & $\begin{array}{c}7.92 \\
\pm 0.3\end{array}$ & $\begin{array}{c}5.28 \\
\pm 0.2\end{array}$ & $\begin{array}{c}0.552 \\
\pm 0.02\end{array}$ & $\begin{array}{c}6.2 \\
\pm 0.3\end{array}$ \\
\hline $\begin{array}{l}\text { Pumpkin } \\
\text { juice }\end{array}$ & $5.1 \pm 0.2$ & - & $\begin{array}{c}0.04 \\
\pm 0.001\end{array}$ & $\begin{array}{c}8.52 \\
\pm 0.3\end{array}$ & $\begin{array}{c}3.52 \\
\pm 0.15\end{array}$ & $\begin{array}{c}0.186 \\
\pm 0.01\end{array}$ & $\begin{array}{c}6.4 \\
\pm 0.3\end{array}$ \\
\hline $\begin{array}{l}\text { unclarified } \\
\text { pureed }\end{array}$ & $6.9 \pm 0.3$ & $6.7 \pm 0.3$ & $\begin{array}{c}0.06 \\
\pm 0.001\end{array}$ & $\begin{array}{c}7.67 \\
\pm 0.3\end{array}$ & $\begin{array}{c}1.76 \\
\pm 0.10\end{array}$ & $\begin{array}{c}0.368 \\
\pm 0.01\end{array}$ & $\begin{array}{c}6.25 \pm \\
0.3\end{array}$ \\
\hline
\end{tabular}

Table 3. Physical and chemical characteristics of drinks on the basis of celery juice with adding extract of walnut

\begin{tabular}{|c|c|c|c|c|c|c|}
\hline Food components & $\mathrm{pH}$ & $\begin{array}{c}\text { Titrated } \\
\text { acids, } \\
\%\end{array}$ & $\begin{array}{c}\text { Soluble } \\
\text { solids, } \\
\%\end{array}$ & $\begin{array}{c}\text { L-anti- } \\
\text { scorbutin, } \\
\mathrm{M \Gamma} / 100 \Gamma\end{array}$ & $\begin{array}{c}\text { Clarity, } \\
\%\end{array}$ & $\begin{array}{c}\text { Clarity, } \\
\text { unit of } \\
\text { optical } \\
\text { density }\end{array}$ \\
\hline $\begin{array}{c}\text { Celery juice : sugar } \\
\text { syrup : walnut extract }\end{array}$ & $\begin{array}{c}5.65 \pm \\
0.2\end{array}$ & $\begin{array}{c}8.5 \pm \\
0.4\end{array}$ & $\begin{array}{c}22.10 \\
\pm 1\end{array}$ & $\begin{array}{c}6.50 \pm \\
0.3\end{array}$ & $83 \pm 3$ & $\begin{array}{c}0.20 \pm \\
0.001\end{array}$ \\
\hline $\begin{array}{c}\text { Celery juice : sugar } \\
\text { syrup: walnut extract: } \\
\text { citric acid }\end{array}$ & $\begin{array}{c}5.00 \pm \\
0.2\end{array}$ & $\begin{array}{c}9.0 \pm \\
0.4\end{array}$ & $\begin{array}{c}21.37 \\
\pm 1\end{array}$ & $\begin{array}{c}5.28 \pm \\
0.3\end{array}$ & $50 \pm 2$ & $\begin{array}{c}0.23 \pm \\
0.001\end{array}$ \\
\hline $\begin{array}{c}\text { Celery juice : honey } \\
\text { solution: walnut } \\
\text { extract }\end{array}$ & $\begin{array}{c}5.25 \pm \\
0.2\end{array}$ & $\begin{array}{c}10.0 \pm \\
0.4\end{array}$ & $\begin{array}{c}23.20 \\
\pm 1\end{array}$ & $\begin{array}{c}5.28 \pm \\
0.3\end{array}$ & $90 \pm 3$ & $\begin{array}{c}0.28 \pm \\
0.001\end{array}$ \\
\hline $\begin{array}{c}\text { Celery juice : honey } \\
\text { solution: walnut } \\
\text { extract }\end{array}$ & $\begin{array}{c}5.70 \pm \\
0.2\end{array}$ & $\begin{array}{c}12.0 \pm \\
0.5\end{array}$ & $\begin{array}{c}22.44 \\
\pm 1\end{array}$ & $\begin{array}{c}7.87 \pm \\
0.3\end{array}$ & $60 \pm 3$ & $\begin{array}{c}0.26 \pm \\
0.001\end{array}$ \\
\hline $\begin{array}{c}\text { Celery juice : honey } \\
\text { solution : walnut }\end{array}$ & $5.60 \pm$ \\
$\begin{array}{c}\text { extract : pepper mint } \\
0.2\end{array}$ & $\begin{array}{c}12.2 \pm \\
0.5\end{array}$ & $\begin{array}{c}21.37 \\
\pm 1\end{array}$ & $\begin{array}{c}7.90 \pm \\
0.3\end{array}$ & $99 \pm 3$ & $\begin{array}{c}0.24 \pm \\
0.001\end{array}$ \\
\hline
\end{tabular}


Selected raw materials - Cornelian cherry and walnut extract of milk-wax maturity stage - as to their chemical composition are considered to be medical and immunomodulating, because they are charachterised by extremely high content of biologically active polyphenols and vitamin $\mathrm{C}$ with a sufficient amount of pectin substances. Technologies of drinks and their physical and chemical characteristics are given in Tables 4 and 5.

Table 4. Physical and chemical characteristics of blended drinks on the basis of juice from Jerusalem artichoke with walnut additive

\begin{tabular}{|c|c|c|c|c|c|c|}
\hline \multirow{2}{*}{$\begin{array}{c}\text { Food } \\
\text { components }\end{array}$} & \multicolumn{3}{|c|}{ Mass content, \% } & \multicolumn{2}{|c|}{$\begin{array}{c}\text { Content by } \\
\text { weight, } \mathrm{mg} / 100 \mathrm{~g}\end{array}$} & \multirow[b]{2}{*}{$\mathrm{pH}$} \\
\hline & $\begin{array}{c}\text { dry } \\
\text { substances }\end{array}$ & $\begin{array}{l}\text { titrated } \\
\text { acids }\end{array}$ & $\begin{array}{c}\text { pectin } \\
\text { substances }\end{array}$ & $\begin{array}{c}\text { L- anti- } \\
\text { scorbutin }\end{array}$ & $\begin{array}{l}\text { poly- } \\
\text { phenols }\end{array}$ & \\
\hline $\begin{array}{c}\text { Jerusalem } \\
\text { artichoke juice : } \\
\text { pureed plum : } \\
\text { sugar extract }\end{array}$ & $\begin{array}{l}21.2 \\
\pm 1.0\end{array}$ & $\begin{array}{c}0.777 \pm \\
0.03\end{array}$ & $\begin{array}{l}0.552 \\
\pm 0.02\end{array}$ & $\begin{array}{c}58.08 \\
\pm 2.0\end{array}$ & $\begin{array}{l}2175 \\
\pm 100\end{array}$ & $\begin{array}{l}4.05 \\
\pm 0.2\end{array}$ \\
\hline $\begin{array}{c}\text { Jerusalem } \\
\text { artichoke juice : } \\
\text { pureed plum : } \\
\text { sugar extract : } \\
\text { ethanolic extract }\end{array}$ & $\begin{array}{l}24.2 \\
\pm 1.1\end{array}$ & $\begin{array}{c}0.536 \pm \\
0.02\end{array}$ & $\begin{array}{l}0.292 \\
\pm 0.01\end{array}$ & $\begin{array}{c}22.88 \\
\pm 1.0\end{array}$ & $\begin{array}{c}1920 \\
\pm 90\end{array}$ & $\begin{array}{l}4.15 \\
\pm 0.2\end{array}$ \\
\hline \begin{tabular}{|c|} 
Jerusalem \\
artichoke juice : \\
apple juice: \\
sugar extract \\
\end{tabular} & $\begin{array}{l}22.2 \\
\pm 1.0\end{array}$ & $\begin{array}{l}0.456 \pm \\
0.02\end{array}$ & $\begin{array}{l}0.173 \\
\pm 0.01\end{array}$ & $\begin{array}{c}66.00 \\
\pm 2.0\end{array}$ & $\begin{array}{c}2070 \\
\pm 90\end{array}$ & $\begin{array}{l}4.70 \\
\pm 0.2\end{array}$ \\
\hline \begin{tabular}{|c|} 
Jerusalem \\
artichoke juice : \\
apple juice : sugar \\
syrup : ethanolic \\
extract \\
\end{tabular} & $\begin{array}{l}25.6 \\
\pm 1.1\end{array}$ & $\begin{array}{l}0.362 \pm \\
0.02\end{array}$ & $\begin{array}{l}0.184 \\
\pm 0.01\end{array}$ & $\begin{array}{c}37.84 \\
\pm 2.0\end{array}$ & $\begin{array}{c}1950 \\
\pm 90\end{array}$ & $\begin{array}{l}4.80 \\
\pm 0.2\end{array}$ \\
\hline $\begin{array}{c}\text { Jerusalem } \\
\text { artichoke juice : } \\
\text { lemon juice: } \\
\text { sugar extract }\end{array}$ & $\begin{array}{l}23.0 \\
\pm 1.0\end{array}$ & $\begin{array}{l}1.340 \pm \\
0.05\end{array}$ & $\begin{array}{l}0.276 \\
\pm 0.01\end{array}$ & $\begin{array}{c}85.38 \\
\pm 4.0\end{array}$ & $\begin{array}{l}2515 \\
\pm 120\end{array}$ & $\begin{array}{l}6.40 \\
\pm 0.2\end{array}$ \\
\hline $\begin{array}{c}\text { Jerusalem } \\
\text { artichoke juice : } \\
\text { lemon juice : } \\
\text { sugar syrup : } \\
\text { ethanolic extract }\end{array}$ & $\begin{array}{l}22.6 \\
\pm 1.0\end{array}$ & $\begin{array}{c}0.437 \pm \\
0.02\end{array}$ & $\begin{array}{l}0.330 \\
\pm 0.01\end{array}$ & $\begin{array}{l}45.76 \\
\pm 2.0\end{array}$ & $\begin{array}{l}1575 \\
\pm 70\end{array}$ & $\begin{array}{l}4.60 \\
\pm 0.2\end{array}$ \\
\hline
\end{tabular}


Table 4 (continued)

\begin{tabular}{|c|c|c|c|c|c|c|}
\hline \multirow{2}{*}{$\begin{array}{c}\text { Food } \\
\text { components }\end{array}$} & \multicolumn{3}{|c|}{ Mass content, $\%$} & \multicolumn{2}{|c|}{$\begin{array}{c}\text { Content by } \\
\text { weight, } \mathrm{mg} / 100 \mathrm{~g}\end{array}$} & \multirow[b]{2}{*}{$\mathrm{pH}$} \\
\hline & $\begin{array}{c}\text { dry } \\
\text { substanc } \\
\text { es }\end{array}$ & $\begin{array}{l}\text { titrated } \\
\text { acids }\end{array}$ & $\begin{array}{c}\text { pectin } \\
\text { substances }\end{array}$ & $\begin{array}{l}\text { L- anti- } \\
\text { scorbutin }\end{array}$ & $\begin{array}{l}\text { poly- } \\
\text { phenols }\end{array}$ & \\
\hline $\begin{array}{c}\text { Jerusalem } \\
\text { artichoke juice } \\
\text { : orange juice : } \\
\text { sugar extract }\end{array}$ & $\begin{array}{l}25.8 \\
\pm 1.1\end{array}$ & $\begin{array}{c}0.50 \\
\pm 0.02\end{array}$ & $\begin{array}{l}0.221 \\
\pm 0.01\end{array}$ & $\begin{array}{c}95.80 \\
\pm 4.0\end{array}$ & $\begin{array}{l}2475 \\
\pm 120\end{array}$ & $\begin{array}{l}5.10 \\
\pm 0.2\end{array}$ \\
\hline $\begin{array}{l}\text { Jerusalem } \\
\text { artichoke juice : } \\
\text { orange juice : } \\
\text { sugar syrup: } \\
\text { ethanolic extract }\end{array}$ & $\begin{array}{l}25.4 \\
\pm 1.1\end{array}$ & $\begin{array}{l}0.375 \\
\pm 0.02\end{array}$ & $\begin{array}{l}0.552 \\
\pm 0.02\end{array}$ & $\begin{array}{c}56.32 \pm \\
2.0\end{array}$ & $\begin{array}{l}2175 \\
\pm 90\end{array}$ & $\begin{array}{l}5.20 \\
\pm 0.2\end{array}$ \\
\hline
\end{tabular}

Using selected materials allowed to combine sour-sweet raw materials and to get interesting gustative and aromatic composition with adding walnut extract and to increase the biological value of Jerusalem artichoke and fruit blends (Table 4).

Table 5. Physical and chemical characteristics of blended drinks on the basis of pureed Jerusalem artichoke with walnut additive

\begin{tabular}{|c|c|c|c|c|}
\hline \multirow{2}{*}{ Food components } & \multicolumn{2}{|c|}{ Mass content, \% } & \multicolumn{2}{c|}{$\begin{array}{c}\text { Content by weight, } \\
\mathrm{mg} / 100 \mathrm{~g}\end{array}$} \\
\cline { 2 - 5 } & $\begin{array}{c}\text { dry } \\
\text { substances }\end{array}$ & $\begin{array}{c}\text { pectin } \\
\text { substances }\end{array}$ & $\begin{array}{c}\text { L-anti- } \\
\text { scorbutin }\end{array}$ & polyphenols \\
\hline $\begin{array}{c}\text { Pureed Cornelian cherry : } \\
\text { pureed Jerusalem artichoke : } \\
\text { sugar syrup (control) }\end{array}$ & $16.8 \pm 0.8$ & $0.26 \pm 0.01$ & $\begin{array}{c}18.6 \\
\pm 0.8\end{array}$ & $252 \pm 10$ \\
\hline $\begin{array}{c}\text { Pureed Cornelian cherry : } \\
\text { walnut extract : sugar syrup }\end{array}$ & $18.6 \pm 0.8$ & $0.31 \pm 0.01$ & $\begin{array}{c}372 \\
\pm 15\end{array}$ & $498 \pm 20$ \\
\hline $\begin{array}{c}\text { Pureed Jerusalem artichoke : } \\
\text { walnut extract : sugar syrup }\end{array}$ & $11.2 \pm 0.5$ & $0.22 \pm 0.01$ & $\begin{array}{c}231.2 \\
\pm 10\end{array}$ & $196 \pm 10$ \\
\hline $\begin{array}{c}\text { Pureed Cornelian cherry: } \\
\text { pureed Jerusalem artichoke : } \\
\text { walnut extract: sugar syrup }\end{array}$ & $14.0 \pm 0.7$ & $0.23 \pm 0.01$ & $\begin{array}{c}372 \\
\pm 15\end{array}$ & $336 \pm 15$ \\
\hline
\end{tabular}

It was confirmed that carrots, pumpkin and semifinished products on their basis and rhubarb juice are rich in sugars, organic acids, $\beta$-carotene and pectin (Table 2). In order to improve the organoleptic properties and to increase food and biological value of drinks blending of carrot or pumpkin juice (puree) with acidic rhubarb juice which was used in order to replace 
citric acid, which is traditionally used in the production, was done. The main physical and chemical indicators of the developed drinks are listed in Tables 6 and 7.

Table 6. Physical and chemical characteristics drinks on the basis of carrots with walnut additive

\begin{tabular}{|c|c|c|c|c|c|c|c|}
\hline \multirow{2}{*}{$\begin{array}{c}\text { Food } \\
\text { components } \\
\text { variants of } \\
\text { recipes }\end{array}$} & \multicolumn{3}{|c|}{ Mass content, $\%$} & \multicolumn{3}{|c|}{$\begin{array}{c}\text { Content by weight, } \\
\mathrm{mg} / 100 \mathrm{~g}\end{array}$} & \multirow[b]{2}{*}{$\mathrm{pH}$} \\
\hline & $\begin{array}{c}\text { dry } \\
\text { substance }\end{array}$ & $\begin{array}{l}\text { titrated } \\
\text { acids }\end{array}$ & $\begin{array}{c}\text { pectin } \\
\text { substances }\end{array}$ & $\begin{array}{c}\text { L-anti- } \\
\text { scorbutin }\end{array}$ & $\begin{array}{c}\beta- \\
\text { carotine }\end{array}$ & \begin{tabular}{|} 
polyphe \\
nols
\end{tabular} & \\
\hline \multicolumn{8}{|c|}{ Carrot juice + rhubarb juice+ sugar syrup (control) } \\
\hline Control & $19.0 \pm 0.8$ & $\begin{array}{l}0.241 \\
\pm 0.01\end{array}$ & $\begin{array}{l}0.298 \\
\pm 0.01\end{array}$ & $\begin{array}{c}1.8 \\
\pm 0.08\end{array}$ & $\begin{array}{l}1.725 \\
\pm 0.06\end{array}$ & $\begin{array}{c}585.0 \\
\pm 25\end{array}$ & \begin{tabular}{|c|}
5.4 \\
\pm 0.2 \\
\end{tabular} \\
\hline \multicolumn{8}{|c|}{ Carrot juice + rhubarb juice + sugar nut extract + sugar syrup } \\
\hline 1 variant & .0 & $\begin{array}{l}0.429 \\
\pm 0.02 \\
\end{array}$ & $\begin{array}{l}0.331 \\
\pm 0.01 \\
\end{array}$ & $\begin{array}{c}51.0 \\
\pm 2 \\
\end{array}$ & \begin{tabular}{|r|}
1.689 \\
\pm 0.06 \\
\end{tabular} & $\begin{array}{c}3787.5 \\
\pm 180 \\
\end{array}$ & $\begin{array}{c}4.7 \\
\pm 0.2 \\
\end{array}$ \\
\hline \multicolumn{8}{|c|}{ Carrot juice + rhubarb juice + alcohol nut extract + sugar syrup } \\
\hline 2 variant & $16.8 \pm 0.8$ & $\begin{array}{l}0.295 \\
\pm 0.01 \\
\end{array}$ & $\begin{array}{l}0.298 \\
\pm 0.01 \\
\end{array}$ & $\begin{array}{r}32.0 \\
\pm 1.5 \\
\end{array}$ & $\begin{array}{l}1.725 \\
\pm 0.06 \\
\end{array}$ & $\begin{array}{c}915.0 \\
\pm 40 \\
\end{array}$ & $\begin{array}{c}5.4 \pm \\
0.2 \\
\end{array}$ \\
\hline \multicolumn{8}{|c|}{ Pureed carrot + rhubarb juice + sugar syrup } \\
\hline Control & $18.0 \pm 0.8$ & $\begin{array}{l}0.616 \\
\pm 0.02 \\
\end{array}$ & $\begin{array}{l}0.662 \\
\pm 0.02 \\
\end{array}$ & $\begin{array}{c}1.65 \\
\pm 0.08 \\
\end{array}$ & \begin{tabular}{|c|}
1.717 \\
\pm 0.06 \\
\end{tabular} & $\begin{array}{l}727.5 \\
\pm 35 \\
\end{array}$ & $\begin{array}{c}4.5 \\
\pm 0.2 \\
\end{array}$ \\
\hline \multicolumn{8}{|c|}{ Pureed arrot + rhubarb juice + alcohol nut extract + sugar syrup } \\
\hline 1 variant & $16.8 \pm 0.7$ & $\begin{array}{l}0.670 \\
\pm 0.02 \\
\end{array}$ & $\begin{array}{l}0.621 \\
\pm 0.02 \\
\end{array}$ & $\begin{array}{c}1.80 \\
\pm 0.08 \\
\end{array}$ & \begin{tabular}{|c|}
1.733 \\
\pm 0.06 \\
\end{tabular} & $\begin{array}{c}835.0 \\
\pm 40 \\
\end{array}$ & \begin{tabular}{c|}
4.2 \\
\pm 0.2 \\
\end{tabular} \\
\hline \multicolumn{8}{|c|}{ Pureed arrot + rhubarb juice + sugar nut extract + sugar syrup } \\
\hline 2 variant & $18.6 \pm 0.8$ & $\begin{array}{l}0.697 \\
\pm 0.02 \\
\end{array}$ & $\begin{array}{l}0.626 \\
\pm 0.02 \\
\end{array}$ & $\begin{array}{l}25.0 \\
\pm 1.0 \\
\end{array}$ & \begin{tabular}{|c|}
1.702 \\
\pm 0.06 \\
\end{tabular} & $\begin{array}{c}3487.5 \\
\pm 150 \\
\end{array}$ & \begin{tabular}{|c|}
4.3 \\
\pm 0.2 \\
\end{tabular} \\
\hline
\end{tabular}

Therefore, to improve the taste of the finished product and to enrich it with vitamins is possible by biologically valuable extracts obtained from walnut of milk-wax maturity stage. The resulting blends on the basis of carrot raw material with adding of sugar extract had a sweet taste with a pleasant acidity, nutty flavor with a slight carrot flavour, color (depending on the number of nut additives) is from dark yellow to brown. In blends of alcohol and water additives nut bitterness was felt.

Blend from raw pumpkin had a pleasant sweet taste, pleasant aroma of pumpkin with slightly noticeable nut color - from orange to dark brown (depending on the number of nut additives). Drinks with pulp had fruit aroma, reminiscent of a mixture of pears, apples and plums. 
Table 7. Physical and chemical characteristics drinks on the basis of pumpkin with walnut additive

\begin{tabular}{|c|c|c|c|c|c|c|c|}
\hline \multirow[b]{2}{*}{$\begin{array}{l}\text { Food components / } \\
\text { variants of recipes }\end{array}$} & \multicolumn{3}{|c|}{ Mass content, $\%$} & \multicolumn{3}{|c|}{$\begin{array}{c}\text { Content by weight, } \\
\mathrm{mg} / 100 \mathrm{~g}\end{array}$} & \multirow[b]{2}{*}{$\mathrm{pH}$} \\
\hline & $\begin{array}{c}\text { dry } \\
\text { substan } \\
\text { ces }\end{array}$ & $\begin{array}{l}\text { titrated } \\
\text { acids }\end{array}$ & \begin{tabular}{|} 
pectin \\
substan \\
ces
\end{tabular} & $\begin{array}{l}\text { L-anti- } \\
\text { scorbutin }\end{array}$ & $\begin{array}{c}\beta- \\
\text { carotine }\end{array}$ & $\begin{array}{l}\text { poly- } \\
\text { phenols }\end{array}$ & \\
\hline $\begin{array}{l}\text { Pumpkin juice }+ \\
\text { rhubarb juice }+ \text { sugar } \\
\text { syrup (control) }\end{array}$ & $\begin{array}{l}20.0 \\
\pm 0.9\end{array}$ & $\begin{array}{c}0.59 \\
\pm 0.02\end{array}$ & $\begin{array}{l}0.552 \\
\pm 0.02\end{array}$ & $\begin{array}{l}7.04 \\
\pm 0.3\end{array}$ & $\begin{array}{r}1.725 \\
\pm 0.06\end{array}$ & $\begin{array}{l}397 \\
\pm 16\end{array}$ & $\begin{array}{c}3.7 \\
\pm 0.1\end{array}$ \\
\hline $\begin{array}{l}\text { Pumpkin juice }+ \\
\text { rhubarb juice }+ \\
\text { alcohol nut extract }+ \\
\text { sugar }\end{array}$ & $\begin{array}{l}21.0 \\
\pm 0.9\end{array}$ & $\begin{array}{c}0.52 \\
\pm 0.02\end{array}$ & $\begin{array}{l}0.541 \\
\pm 0.02\end{array}$ & $\begin{array}{l}17.60 \\
\pm 0.8\end{array}$ & $\begin{array}{l}1.749 \\
\pm 0.06\end{array}$ & $\begin{array}{r}563 \\
\pm 25\end{array}$ & $\begin{array}{c}3.7 \\
\pm 0.1\end{array}$ \\
\hline $\begin{array}{l}\text { Pumpkin juice }+ \\
\text { rhubarb juice }+ \text { sugar } \\
\text { nut extract } \\
+ \text { sugar }\end{array}$ & $\begin{array}{r}13.8 \\
\pm 0.6\end{array}$ & $\begin{array}{l}0.31 \\
\pm 0.01\end{array}$ & $\begin{array}{l}0.515 \\
\pm 0.02\end{array}$ & $\begin{array}{l}14.08 \\
\pm 0.6\end{array}$ & $\begin{array}{l}1.705 \\
\pm 0.06\end{array}$ & $\begin{array}{l}1050 \\
\pm 50\end{array}$ & $\begin{array}{c}3.8 \\
\pm 0.1\end{array}$ \\
\hline $\begin{array}{l}\text { Pumpkin juice }+ \\
\text { rhubarb juice }+ \text { sugar } \\
\text { nut extract }+ \text { honey } \\
\text { syrup }\end{array}$ & $\begin{array}{r}19.2 \\
\pm 0.8\end{array}$ & $\begin{array}{c}0.36 \\
\pm 0.01\end{array}$ & $\begin{array}{l}0.520 \\
\pm 0.02\end{array}$ & $\begin{array}{l}15.84 \\
\pm 0.6\end{array}$ & $\begin{array}{l}1.712 \\
\pm 0.06\end{array}$ & $\begin{array}{l}1125 \\
\pm 50\end{array}$ & $\begin{array}{l}3.65 \\
\pm 0.1\end{array}$ \\
\hline $\begin{array}{l}+ \text { rhubarb juice ciк }+ \\
\text { alcohol nut extract }+ \\
\text { honey syrup }\end{array}$ & $\begin{array}{r}15.0 \\
\pm 0.7\end{array}$ & $\begin{array}{c}0.40 \\
\pm 0.01\end{array}$ & $\begin{array}{l}0.532 \\
\pm 0.02\end{array}$ & $\begin{array}{l}11.26 \\
\pm 0.5\end{array}$ & $\begin{array}{l}1.703 \\
\pm 0.06\end{array}$ & $\begin{array}{r}563 \\
\pm 25\end{array}$ & $\begin{array}{l}3.60 \\
\pm 0.1\end{array}$ \\
\hline $\begin{array}{l}\text { Pureed pumpkin }+ \\
\text { rhubarb juice }+ \text { sugar } \\
\text { (control) }\end{array}$ & $\begin{array}{l}23.2 \\
\pm 1.0\end{array}$ & $\begin{array}{c}0.28 \\
\pm 0.01\end{array}$ & $\begin{array}{l}0.461 \\
\pm 0.02\end{array}$ & $\begin{array}{l}7.73 \\
\pm 0.3\end{array}$ & $\begin{array}{l}1.712 \\
\pm 0.06\end{array}$ & $\begin{array}{l}548 \\
\pm 25\end{array}$ & $\begin{array}{c}3.7 \\
\pm 0.1\end{array}$ \\
\hline $\begin{array}{l}\text { Pureed pumpkin }+ \\
\text { rhubarb juice }+ \\
\text { alcohol nut extract }+ \\
\text { sugar syrup }\end{array}$ & $\begin{array}{l}23.5 \\
\pm 1.0\end{array}$ & $\begin{array}{c}0.34 \\
\pm 0.01\end{array}$ & $\begin{array}{l}0.342 \\
\pm 0.01\end{array}$ & $\begin{array}{l}9.94 \\
\pm 0.5\end{array}$ & $\begin{array}{r}1.654 \\
\pm 0.06\end{array}$ & $\begin{array}{r}713 \\
\pm 30\end{array}$ & $\begin{array}{l}3.70 \\
\pm 0.1\end{array}$ \\
\hline $\begin{array}{l}\text { Pureed pumpkin }+ \\
\text { rhubarb juice }+ \text { sugar } \\
\text { nut extract }+ \text { sugar } \\
\text { syrup }\end{array}$ & $\begin{array}{l}21.0 \\
\pm 0.9\end{array}$ & $\begin{array}{c}0.38 \\
\pm 0.01\end{array}$ & $\begin{array}{r}0.592 \\
\pm 0.02\end{array}$ & $\begin{array}{c}17.26 \\
\pm 0.8\end{array}$ & $\begin{array}{r}1.755 \\
\pm 0.06\end{array}$ & $\begin{array}{r}810 \\
\pm 30\end{array}$ & $\begin{array}{l}3.70 \\
\pm 0.1\end{array}$ \\
\hline $\begin{array}{l}\text { Pureed pumpkin }+ \\
\text { rhubarb juice }+ \text { alcoho } \\
\text { nut extract }+ \text { honey } \\
\text { syrup }\end{array}$ & $\begin{array}{l}17.0 \\
\pm 0.7\end{array}$ & $\begin{array}{c}0.31 \\
\pm 0.01\end{array}$ & $\begin{array}{r}0.557 \\
\pm 0.02\end{array}$ & $\begin{array}{l}13.25 \\
\pm 0.6\end{array}$ & $\begin{array}{r}1.782 \\
\pm 0.06\end{array}$ & $\begin{array}{r}900 \\
\pm 40\end{array}$ & $\begin{array}{c}3.75 \\
\pm 0.1\end{array}$ \\
\hline
\end{tabular}

It is proved that the use of biologically valuable walnut supplements favorably affects not only the physical and chemical properties, but the organoleptic properties of drinks. Given that sugar nut extract on the basis of 
a mellow, it is possible to increase the content of the recipe of blends doubled compared to the water-alcohol.

\section{CONCLUSIONS}

Research has demonstrated that celery, Jerusalem artichoke, carrots, pumpkin, and rhubarb are valuable raw materials for new fruit and vegetable drinks. Using nut additives with walnut of milk-wax maturity stage in blends allows enriching their biologically active substances and creating original compositions for flavor. The technologies of drinks can be recommended for use in daily human diet in order to enrich the body by biologically valuable components.

\section{REFERENCES}

1. Arasymovych, V.V., Baltaha, S.V., Panomareva, N.P. (1990). Methods of analyzing pectin substances, hemicelluloses and pectinolytic enzymes in fruits. Chisinau, Moldavia: RIO AN MSSR. (in Russian)

2. Peresichnyi, M.I., Mazaraki, A.A., Kravchenko M.F. et al. (2012).Technology of functional foods (Monograph). Kyiv, Ukraine: National. University of Trade and Economics. (in Ukrainian)

3. Sarnitsky, P.L., Vydrin, Yu.V., Chumachenko, I.P. (2007). Nontraditional forage crops. Kyiv, Ukraine: "Urozhai". (in Russian)

4. Tiurikova, I.S. (2012). Technological aspects of production of extracts from walnuts of milk-wax maturity stage. Equipment and technology of food production. Temat. collection of scientific works (pp.63-69). Donetsk, Ukraine: DonNUET, 28 (in Ukrainian)

5. Tiurikova, I.S. (2013). A way of producing the extract of walnut of milkwax maturity stage. Ukrainian. Patent № 77238. Kyiv: State Intellectual Property Service of Ukraine. (in Ukrainian)

6. Tiurikova, I.S. (2014). A way of producing the dietary supplement of of milk-wax maturity stage. Ukrainian. Patent № 88192. Kyiv: : State Intellectual Property Service of Ukraine. (in Ukrainian)

7. Tiurikova, I.S., Rybak, G.M., Kholodnyi, L.P. (2008). Using fruit green walnuts for high quality products. Food Technology-2008: IV International scientific and practictical conference, 9-10 October, 2008 (pp.168-171). Odessa, Ukraine: ODAFT, 34 (2). (in Ukrainian)

8. Tiurikova, I.S., Rybak, G.M., Kholodnyi, L.P. (2008). Wallnut of milkwax maturity stage - sourse BAR. Equipment and technology of food production. Temat. collection of scientific works (pp.53-57). Donetsk, Ukraine: DonNUET, 18 (in Ukrainian) 
9. Tiurikova, I.S. (2013). Development of non-alcoholic beverages with extracts of green walnuts. Education, Science and Modern Society: Current Issues in Economics and Cooperation: International scientific and practical conference of professor and lecturer staff and graduate students, 16-17 April, 2013. (pp. 243-250). Belgorod, Russia: BUKEP. (in Russian).

10. Tiurikova, I.S. (2013) Using the celery and green walnut to create functional purpose drinks. Science. Education. Youth: The Republican scientific and practical Conference of Young Scientists, 18-19 April, 2013. (pp. 93-95). Almaty, Kazakhstan: ATU, 2013. (in Russian) 